\title{
ILCEA
}

Revue de l'Institut des langues et cultures

d'Europe, Amérique, Afrique, Asie et Australie

$13 \mid 2010$

Les voies incertaines de la démocratisation

\section{Le récit testimonial en Colombie : une forme de réparation?}

\section{Marie Estripeaut-Bourjac}

\section{OpenEdition \\ Journals}

\section{Édition électronique}

URL : http://journals.openedition.org/ilcea/909

DOI : 10.4000/ilcea.909

ISSN : 2101-0609

Éditeur

UGA Éditions/Université Grenoble Alpes

\section{Édition imprimée}

ISBN : 978-2-84310-181-6

ISSN : 1639-6073

\section{Référence électronique}

Marie Estripeaut-Bourjac, "Le récit testimonial en Colombie : une forme de réparation ? », ILCEA [En ligne], 13 | 2010, mis en ligne le 30 novembre 2010, consulté le 19 avril 2019. URL : http:// journals.openedition.org/ilcea/909; DOI : 10.4000/ilcea.909

Ce document a été généré automatiquement le 19 avril 2019

(c) ILCEA 


\title{
Le récit testimonial en Colombie : une forme de réparation?
}

\author{
Marie Estripeaut-Bourjac
}

1 La spécificité de la situation colombienne, où la Loi Justicia y Paz du 23 juin 2005 «[...] est sans doute le thème d'actualité le plus important dans le domaine de la justice transitionnelle » (Cruvelier, 2008, p. 21) ${ }^{1}$, permettra d'apporter des éléments dans l'étude de cette dernière. Cette communication propose ainsi d'aborder l'ébauche d'une étape de transition dans le conflit colombien à la lumière de l'intense circulation et de l'abondante publication de récits à la première personne qui s'observent aujourd'hui dans le pays.

Des thèmes comme réconciliation et mémoire, vérité, justice, réparation, pardon et victimes, se trouvent en effet actuellement au centre des débats en Colombie. Ils sont le produit d'une pratique testimoniale spécifique et répondent à l'impérieux besoin éprouvé par les victimes d'être écoutées et crues. Mais ils correspondent aussi à une initiative de la société civile, visant à créer l'espace commun d'un vécu partagé et, à plus long terme, à reconstruire un espace public par le débat. Ce phénomène est d'une ampleur telle que les buts de la Loi Justicia y Paz (favoriser la démobilisation des groupes paramilitaires en leur accordant une quasi-impunité) sont aujourd'hui modifiés et dépassent les intentions premières du gouvernement, en raison de la participation active de la société civile, qui est parvenue à ouvrir une brèche dans la version officielle et hégémonique des faits.

La notion de témoignage qui est ici utilisée renvoie essentiellement à une écriture, composée de témoignages directs, d'autobiographies et de récits de vie. Ces récits à la première personne, tels qu'ils sont utilisés en Colombie, constituent une stratégie de justice restauratrice, une élaboration d'un nouveau récit national de la violence et une proposition de paix par la reconnaissance mutuelle. Ils font en outre office de réparation symbolique et de thérapie sociale, menées par ceux qui survivent au milieu de cette guerre irrégulière. 


\section{Les forces en jeu}

4 Les modèles d'autres expériences (comme les Balkans ou l'Afrique centrale) ne peuvent servir à rendre compte des caractéristiques de la violence en Colombie: elle n'est pas le produit d'affrontements politiques, culturels, ethniques, religieux ou sociaux et l'absence de l'État dans de grandes zones du territoire a permis son expansion comme seule voie de résolution des conflits. La dispersion et la durée de cette guerre non reconnue, ses différences régionales, ainsi que les multiples acteurs armés auxquels la population est confrontée (guérilleros, paramilitaires, armées privées, organismes de sécurité de l'État, bandes urbaines, organisations des cartels de la drogue), complètent ces caractéristiques.

Ce conflit, qualifié par certains analystes comme la plus grande crise humanitaire que l'Amérique latine ait connue, provoquant le déplacement de 4 millions de personnes à l'intérieur du pays, 5000 séquestrés, 20000 disparus et 15000 victimes recensées, n'est pas une guerre dans laquelle sont repérables des lignes de front, en raison du caractère fluctuant des territoires occupés par les divers acteurs. Les coopérations économiques établies régionalement entre les uns et les autres débouchent en effet sur des accords locaux instables. Ce n'est pas non plus une guerre civile au sens classique du terme, dans laquelle la population civile adhérerait à une représentation "ami-ennemi ", mais une "guerre contre la société» (Pécaut, 2005, p.4). Il ne s'agit pas de convaincre la population, mais de l'obliger à se soumettre ou à fuir. Le recours à l'intimidation, à la terreur et à la « logique de protection » constituent donc des stratégies centrales et font de « l'emprise locale sur cette population [...] un enjeu majeur du conflit » (Pécaut, 2008, p. 111). Les idéaux sont relégués à l'arrière-plan, pour laisser place à un " prosaïsme » et à une banalisation de la violence, dans lesquels transactions et massacres coexistent et où homicides et enlèvements sont perçus comme étant dans la nature des choses et paramètrent la vie quotidienne. Ces manifestations hétérogènes empêchent ainsi une description d'ensemble de ce conflit. Sa constance, c'est son atrocité, qui, en raison de la prégnance de la religion, relève fréquemment du sacrilège, comme ces massacres, où l'acharnement des paramilitaires sur les corps s'unit à l'outrage (canettes de bière incrustées dans les cadavres ou têtes servant de ballon de football).

6 Le phénomène des desplazados constitue en outre la version contemporaine d'une situation endémique et ne relève pas du seul conflit armé : «Un des recours habituels pour dissimuler cette situation consiste à la présenter comme s'il s'agissait du résultat de l'affrontement entre deux acteurs récents de la violence: la guérilla et les paramilitaires.» (Molano, 2003, p.4.) Le desplazamiento, «vécu, selon Daniel Pécaut, comme une condition sociale presque permanente », prolonge donc une histoire bien plus ancienne et constitue un autre des traits spécifiques de la situation colombienne.

7 Cette guerre, qui permet à certains acteurs d'obtenir plus de bénéfices que dans une situation de paix, se caractérise aujourd'hui par son installation au cœur de l'économie : «La très longue durée de notre conflit est liée à son articulation très particulière à l'économie civile. » (Salazar et Castillo, 2001, p. 110.) Cette dernière assume en effet de façon passive les coûts du conflit, qui absorbe partie de ses excédents. L'absence de toute idéologie et d'un clivage clair dans la population se doit à ce que la plupart des affrontements locaux ne répondent pas à des revendications, mais à des opportunités de "prédation" dans des zones qui dépendent de l'exportation des matières premières. L'accusation portée à la guérilla de s'être « détériorée » n'est en réalité que le symptôme 
«[...] d'une dégradation plus générale qui affecte les institutions et de larges secteurs de la société. Plus que jamais, l'économie de la drogue et ses effets se retrouvent au cœur de la problématique colombienne » (Pécaut, 2008, p. 152).

8 L'actuel repli territorial des $\mathrm{FARC}^{2}$ ne se doit pas à l'efficacité des forces armées, mais plutôt à l'action des paramilitaires, dont les divers groupes étaient unis jusqu'en 2003 par un projet de reconquête territoriale aux dépens de la guérilla. Ils ne combattent pas l'État ni n'affrontent la force publique, mais cherchent à se les concilier et à les infiltrer. Ils bénéficient en effet de leur complaisance, car ils font le « sale boulot » dans cette « guerre sale ». La première élection d'Álvaro Uribe (2002) marque cependant un tournant. Il est le premier président à ne pas envisager de négociation avec les FARC: son objectif prioritaire est la "sécurité démocratique », qui consiste à renforcer l'effort militaire afin d'en finir avec la guérilla sans toucher aux organisations des paramilitaires et des trafiquants de drogue, désormais étroitement imbriqués. Uribe contrôle aussi la classe politique car, pour diverses raisons, les partis sont profondément divisés. La question des paramilitaires a en effet causé une polarisation de l'opinion et ceux qui les dénoncent sont fréquemment soupçonnés de complicité avec la guérilla : «Le gouvernement d'Uribe va surfer sur cette polarisation. » (Pécaut, 2008, p. 106). L'approbation de l'opinion tourne ainsi autour des $70 \%$ durant son premier mandat et va jusqu'à plus de $80 \%$ durant le second, surtout durant la crise avec l'Équateur et le Venezuela en 2008.

9 Les ramifications innombrables de l'économie de la drogue ne se réduisent cependant pas au seul conflit, car si Carlos Castaño ${ }^{3}$ a admis qu'à leur début les AUC étaient financées à $70 \%$ par le narcotrafic, à partir de 2002, elles sont passées à la solde des gros narcotrafiquants. Le pacte de Ralito (2001) révèle en outre leurs liens avec la classe politique : plus de $30 \%$ des représentants seraient élus avec leur soutien, ce qui a donné lieu au scandale de la "para-politique » et à une crise politique générale. En 2008, on comptait une trentaine de parlementaires sous les verrous et cinquante autres mis en examen.

10 La Loi Justicia y Paz, si elle ne reconnaît pas les paramilitaires comme acteurs politiques, les fait «[...] pourtant bénéficier d'un traitement politique [...] qu'ils doivent aux massacres et autres atrocités qu'ils ont perpétrés. La cruauté est bien ce qui ouvre en certaines circonstances la voie vers le politique» (Pécaut, 2008, p. 131). Cette loi, qui « dicte les dispositions pour la réinsertion de groupes armés fonctionnant hors du cadre légal ", mais qui de fait, offre l'amnistie et une quasi-impunité aux groupes paramilitaires qui acceptent de retourner à la vie civile, a bouleversé de nombreux secteurs de la société. Justicia y Paz n'a pas en effet été pensée en fonction des victimes, mais des criminels et à ce qu'ils sont disposés à accepter pour être « réintégrés » dans la société. Cette loi signifie en outre la réinsertion de 40000 démobilisés, sur laquelle il y a très peu d'information. Pour beaucoup, dans cette loi, il n'y a donc ni justice ni paix, car il n'y est pas question de vérité.

11 Ainsi, l'historienne María Victoria Uribe juge urgent de répondre à « Pourquoi tout cela est-il arrivé ?4 », mais rappelle que la vérité historique ne se substitue pas à la vérité juridique. Celle-ci n'est pas binaire (coupable/victime), mais cherche à établir les responsabilités collectives, afin de faciliter la réparation et «metttre fin à une mémoire qui se transmet de génération en génération ». Javier Darío Restrepo affirme qu'« une réconciliation sans vérité est une blessure mal refermée ${ }^{5}$ ", tandis que l'historien Gonzalo Sánchez souligne l'urgence d'une thérapie sociale, afin de pouvoir, un jour, «Pardonner l'impardonnable » (entrevue avec l'auteur, 21 juin 2005). 
12 Discuter de la question des réparations en plein conflit représente un défi. Il est en effet impossible de déterminer le nombre, toujours croissant de victimes, car de nouvelles violences menacent d'entraver d'éventuels processus de restitution. La Loi Justicia y Paz présente ainsi une situation inédite, limitant les comparaisons avec d'autres processus de justice transitionnelle. Elle se réalise en effet dans un régime démocratique et ne s'applique pas a posteriori, mais en pleine guerre et en présence d'acteurs armés qui n'ont pas été vaincus et dont seulement une partie est invitée à négocier. Cette loi se discute aussi en présence d'une presse forte et professionnelle - ce qui la différencie de la plupart des pays où des tribunaux internationaux ont été installés -, et face à une communauté internationale, qui a accepté hier l'impunité comme le coût du rétablissement de la paix ou de la démocratie, mais qui aujourd'hui n'est plus disposée à de telles concessions.

Pour Thierry Cruvelier, il s'agit «[...] d'un système indulgent de justice criminelle domestique, qui met l'accent sur la revélation de la vérité et l'indemnisation [...]» (Cruvelier, 2008, p. 21). Cependant, cette loi « [...] a montré sa sensibilité à la fluctuante ambiance politique colombienne " (ibid., p. 21) et a connu des changements significatifs en cours de route. En 2008, le premier résultat des confessions des paramilitaires a été l'identification de centaines de fosses communes, contenant plus de 2000 corps. Sergio Jaramillo estimait ainsi en 2005 que l'aspect positif de la Loi Justicia y Paz était qu'après de telles révélations, il sera désormais impossible d'ignorer « les victimes, jusqu'à présent les fantômes du conflit » (Jaramillo, 2005, p. 6).

\section{La valeur symbolique du récit testimonial}

L'histoire de la littérature colombienne est traversée par la violence et, selon Karl Kohut, c'est là sa caractéristique principale, comparée à celle des autres pays du continent (Kohut, 1994). Chaque génération a en effet été confrontée à cette violence, mais la Colombie est cependant représentée de façon récurrente comme étant frappée d'amnésie: «Rien ne durait, rien ne se perpétuait dans ce monde sans mémoire ni passé. » (Moreno, 1987, p. 220.) Ces dernières années, on observe toutefois un changement dans l'appréhension par l'écriture de cette guerre incessante aux multiples acteurs. Elle est en effet envisagée aujourd'hui directement, à partir de l'expérience et du vécu au quotidien de ses acteurs, victimes ou témoins, et incombe désormais à un « je », qui révèle comment la violence envahit jusqu'au plus intime de l'être et le détériore psychiquement. Ainsi, Stella, ex-délinquante devenue guérillera : «Je ne suis pas méchante. Je faisais tout ça parce que la vie m'a obligée à le faire, mais pas parce que je le voulais. » (González Uribe, 2002, p. 66.) Gloria Posada décrit, quant à elle, son quartier à Medellín :

\footnotetext{
Nous avons entendu les premiers affrontements il y a deux ans [...]. Nous n'aurions jamais imaginé qu'au cours des mois suivants, l'explosion de grenades, le bourdonnement des balles, les bombes qui éclatent, la fumée, deviendraient un paysage quotidien, diurne et nocturne, une alarme pour se réveiller au petit matin, un signal pour se barricader chez soi, un questionnement sur s'en aller et tout abandonner, ou rester et attendre le cours des heures.

[...] Grimper la côte du lotissement devint un acte de peur pour la survie [...]. (Posada, 2006, p. 2-3.)
}

L'ampleur du phénomène testimonial colombien révèle l'existence d'un «mouvement social» (Dulong, 2003, p. 11), dont la dimension civile s'oppose à la valorisation de la figure du guerrier, qui a prévalu jusqu'à présent. Rappelons que cette écriture a constitué «la forme narrative la plus prépondérante associée aux révolutions d'Amérique 
centrale» (Beverley et Zimmerman, 1990, p. XI). En Colombie, cette démarche s'inscrit dans une réflexion générale sur un possible processus collectif de réconciliation, dans lequel la vérité et la mémoire sont les atouts fondamentaux pour envisager une amnistie qui pardonne, mais n'oublie pas.

La première visée de cet ensemble d'écrits consiste à construire une mémoire plus longue, car si « la terreur impose l'oubli » (Arendt, 1972, p. 180), elle rompt aussi les espaces de socialité, interdit les déplacements et les échanges. Il s'agit ensuite de produire des connaissances sur le conflit, en faisant appel à d'autres sources. Par la méthodologie du récit de vie, A. Molano cherche ainsi à " [...] ne plus faire de la Violence une abstraction » (Molano et Ramírez, 1985, p. 29) et à révéler qu'elle est « [...] un microorganisme niché dans les entrailles sociales de la Colombie» (Angulo, 1985, p. 19). Ainsi, Don Rafael, membre des Autodéfenses: "Celle qui ne m'a jamais laissé tomber et est venue me chercher partout, c'est cette maudite violence, celle-là est vraiment plus fidèle qu'une fiancée laide. » (Salazar, 1999, p. 67.) L'écoute de l'autre, acteur ou victime, à l'œuvre dans la pratique testimoniale, permet de dégager des narrations opposées, fondamentales dans ce cadre de guerre irrégulière. Elles révèlent en effet une dialectique victime/victimaire, permettant de mettre en consonance les représentations individuelles avec une symbolique collective partagée, au-delà des affrontements. Cette nouvelle façon d'aborder le conflit s'effectue donc à partir de ce qui caractérise l'être humain, son aptitude à verbaliser et à symboliser :

Le conflit colombien est aussi un affrontement de récits. Les témoignages de l'État, des victimaires, des moyens de communication et de l'académie ont gagné pour l'instant. Pour l'instant, le pays de la dignité de ceux qui ne combattent pas, du sujet collectif qui a survécu au milieu de la guerre et qui [...] a privilégié son rôle de citoyen sur celui du guerrier, pour l'instant ces histoires ne sont pas parvenues à faire partie du grand récit national de la violence. (Franco et Rincón, 2007, p. 5.)

La mise en récit de ce passé qui ne passe pas, en nommant l'innommable, ouvre un univers symbolique et sémantique à quelque chose qui n'a pas été exprimée jusqu'alors. Il «transforme cette violence en discours » (Uribe, 2004, p. 79) et établit un dialogue et une cohabitation entre des secteurs qui s'auto-représentent comme sans communication possible. Sous forme de bribes et de fragments, la pratique testimoniale construit ainsi un de « ces espaces de médiation [qui] ont eu un rôle essentiel dans l'histoire » (Gruzinski, 1999, p. 43) et fait émerger des ensembles de trajets existentiels communs, qui réfractent les circonstances d'une Colombie morcelée entre différents acteurs et lieux de pouvoir. Un projet précis sous-tend cependant ces récits, la réconciliation :

La réconciliation nationale, la paix ne sont pas des signatures, un décret ou une déclaration politique, mais le parcours de tout un peuple blessé et sacrifié, qui offre [...] son pardon, qui connaît son histoire et les responsables de cette dernière et qui demande réparation et justice. (Santamaria, dans Franco et Rincón, 2007, p. 5.)

18 Tout programme de réparation, selon Pablo de Greiff, doit se donner des buts précis, car son efficacité réside dans sa cohérence avec des sorties de conflit privilégiant la justice (y compris la justice pénale) et la vérité. Une véritable politique de justice transitionnelle doit donc être accompagnée d'élucidation de l'histoire et/ou de réforme institutionnelle. Il faut premièrement ne pas se contenter de reconnaître son statut à la victime, mais aussi la reconnaître en tant que citoyen, et donc ne pas «[...] consolider l'identité des victimes en tant que victimes mais à l'inverse, les motiver à s'identifier à nouveau ou pour la première fois, en tant que citoyens bénéficiant des même droits que les autres " (de Greiff, 2005, p. 11). Dans le cas de la Colombie, la pratique testimoniale est un moyen 
de réparation, dont la forme participative permet la réappropriation d'une citoyenneté et la recréation, au-delà de l'État, d'une confiance civique dans certaines institutions

Inciter les victimes à "avoir confiance dans les institutions de l'État» (ibid., p. 11) constitue donc la seconde visée d'un programme de réparation, car des compensations ne sont pas suffisantes «[...] pour rétablir le lien de confiance entre les citoyens et les institutions qui ne les ont jamais prises en compte ou qui, effectivement ont abusé de leurs droits" (ibid.). Le grand nombre de victimes et d'exactions se superposant aux individus et aux faits et les transformant en une masse anonyme (les déplacés, les disparus), la réparation doit ainsi être liée à une conception de la reconnaissance comme processus, dans lequel l'individu dont il s'agit de reconnaitre la souffrance est impliqué directement en tant qu'être singulier.

À ces objectifs de reconstruction, mais aussi de justice et de vérité, répond la publication du $\mathrm{GMH}^{6}$, consacrée au massacre de Trujillo (1990, département du Valle), première initiative d'élaboration d'une mémoire historique du conflit armé en Colombie. Elle «[...] se fonde sur la conviction qu'il est possible d'établir un lien indestructible entre la construction de mémoires et la construction démocratique » (Sánchez, 2008, p. 27). Cette publication revendique le caractère politique de la mémoire : lieu de lutte entre diverses versions du passé et choix d'un futur, mais aussi de l'engagement des plus humbles et des plus exclus en tant que sujets politiques. La mémoire se construit en effet de façon asymétrique : les Indigènes et les paysans ne se trouvent pas sur un pied d'égalité avec les élites et les victimes ne bénéficient pas des mêmes moyens que les victimaires pour dire leur vérité. Pour le GMH, la mémoire historique joue un rôle essentiel dans des situations de conflit : « Un des thèmes du débat dans une transition, aussi précaire soit-elle, consiste précisément dans l'évaluation du passé traumatique et des responsabilités sociales, institutionnelles, voire criminelles. »(Ibid., p. 27.) Cependant, la mémoire historique n'est en aucun cas un substitut de la justice, elle en est une forme, lorsque qu'il y a déficit de vérité judiciaire, c'est elle qui « devient le nouveau juge » (ibid., p. 28).

21 La fonction symbolique de l'écriture testimoniale va cependant plus loin. Sa force institutionnelle et son rôle de lien social résident dans son aspect humain, par la confiance qu'elle accorde à la parole d'autrui, laquelle renforce «[...] la similitude en humanité des membres de la communauté. L'échange des confiances spécifie le lien entre des êtres semblables»(Ricœur, 2000, p. 207). Recueillir et produire des témoignages signifie être convaincu que cette "similitude en humanité » a valeur de restauration des valeurs collectives.

22 Cette écriture est en effet témoignage, c'est-à-dire crédibilité accordée à une parole, dont P. Ricœur a rappelé le sens juridique et la valeur sociale. Cette structure stable de la disposition à témoigner la pose comme facteur de sûreté dans les rapports sociaux et « [... ] fait du monde social un monde intersubjectivement partagé. Ce partage est la composante majeure de ce qu'on peut appeler le "sens commun" " (ibid., p. 207). Ce dernier est affecté lorsqu'un pouvoir instaure un climat de surveillance mutuelle et de délation, ou que des pratiques mensongères détruisent la confiance dans la parole. C'est là toute l'importance de cette écriture dans des situations paroxystiques, comme ce fut le cas en Argentine, au Chili et, aujourd'hui, en Colombie.

La diversité sociale et la choralité particulières à la forme testimoniale symbolisent ainsi la reconstruction ou l'établissement d'un lien social et de nouveaux pactes de confiance, particulièrement entre intelligentsia et classes populaires (un médiateur lettré recueille 
et réélabore le récit). Cette forme interactive permet aux partenaires de collaborer par leurs questions et leurs réactions et pose que «l'état du témoin est fonction du crédit accordé, d'emblée et souvent, à un individu préalablement à son récit » (Dulong, 1998, p.15). Cet assentiment naturel dans la parole d'autrui place ainsi la confiance et la sincérité des partenaires au fondement des rapports sociaux, en tant que condition de toute interaction.

\section{Perspectives}

Le cas de la Colombie démontre clairement l'opposition qui peut exister entre les intérêts d'un gouvernement et ceux de la société civile. Christian Tomuschat s'étonne ainsi qu'on y parle de Justice, de Vérité et de Réparation, alors que d'autres ont placé la vérité en premier lieu : «La paix ne peut se construire que sur la base de la vérité; si on ne sait pas ce qui s'est passé, il est probable que l'on commette dans le futur les mêmes erreurs qui ont déclenché le conflit. » (Tomuschat, 2005, p. 15.) La justice constitue ensuite le second pilier de la paix, car il est inimaginable de tout pardonner et amnistier et de laisser l'assassin d'hier devenir un homme libre du jour au lendemain, ce serait «[...] nier le fondement de la morale et de la confiance publique » (ibid., p. 15). S'il y a des compromis possibles, «[...] jusqu'à quel point un État de Droit peut-il ignorer son passé criminel, sans affecter les bases d'une coexistence sociale pacifiée dans le futur? » (ibid., p. 15).

Selon Eduardo Pizarro ${ }^{7}$, à partir de 1989, la justice pénale et la justice rétributive (équivalence entre délit et peine) ont été remplacées par d'autres formes dans la négociation de sortie de conflits armés, telles la réparation symbolique et/ou matérielle. En Amérique latine, « la transition vers la démocratie ou le dépassement des séquelles de la guerre civile a été le critère d'approche du thème de la justice » (Pizarro, 2005, p. 2). En ce point, la pratique testimoniale constitue autant un élément indispensable dans un processus de justice transitionnelle qu'une modalité de justice restauratrice, rappelant la catharsis collective permise par le récit public en Afrique du Sud. La publication sur le massacre de Trujillo revêt ainsi une symbolique de tout premier ordre, car elle propose, au-delà de l'attitude gouvernementale, la première mise en forme officielle et collective de l'intense activité testimoniale qui parcourt le pays.

Dans le cas de la Colombie, il vaut mieux parler d'un processus de transition assumé par la société civile, l'État se refusant à reconnaitre son implication dans le conflit et à appliquer une politique générale de justice transitionnelle, qui unirait un programme de réparation à la justice et à l'élucidation de l'histoire. Toute réparation doit en effet s'effectuer au détriment du patrimoine de l'agresseur et, réparer avec des biens confisqués, «[...] sans qu'il y ait une médiation symbolique de la part de l'état peut dénaturer le concept même de réparation ${ }^{8}$ (VV. AA., 2005, p. 23).

Il faut reconnaître toutefois qu'«[i]l se passe beaucoup de choses en Colombie aujourd'hui. Et l'une des plus importantes c'est que, malgré le poids de la peur, les victimes, la société et les institutions ont commencé à parler » (Sánchez, 2008, p. 29). La pratique testimoniale joue un rôle fondamental dans ce changement dans ce contexte si peu propice. Même si les victimaires ont tendance à rationaliser leurs actes au lieu de demander pardon, leurs déclarations favorisent la participation politique et le débat sur la violence, en particulier celle de l'État. C'est ce que Leigh A. Payne nomme «[...] la coexistence contentieuse ou exercice discursif et conflictuel à la démocratie dans des sociétés profondément divisées » (Payne, 2008, p. 8). 

premier devoir de citoyenneté” " (Ackerman, dans Payne, 2008, p. 13). Les assassins parlent parfois contre leur propre intérêt et leur parole crée un conflit politique, qui est « en grande partie discursif» (Payne, 2008, p. 8). En outre, « [...] au lieu de défendre les buts élevés et incertains du consensus ou de la réconciliation, la coexistence contentieuse se base simplement sur un débat ouvert et démocratique" (ibid., p.9) et aucune démocratie ne peut courir le risque de l'éliminer. Les affrontements sur le passé ne cessent pas, mais les adversaires apprennent à vivre ensemble - à coexister - « avec leurs différences irréconciliables dans des démocraties imparfaites» (ibid., p. 14). La coexistence contentieuse est cependant limitée en ce qui concerne les droits de l'homme : elle rend « [...] possible la lutte pour les idéaux démocratiques ; mais elle ne garantit pas [...] son succès » (ibid., p. 14).

Dans l'espace ouvert par le témoignage, qu'il soit réalisé devant des juges ou recueilli par un médiateur, les confessions des assassins perturbent le silence et les contre-vérités. Horacio Verbitsky rappelle ainsi qu'en Argentine, c'est seulement lorsque le capitaine Scilingo a avoué en 1995 «j'ai jeté 30 personnes à la mer », qu'une grande partie de la société a compris qu'il n'y avait pas deux versions de l'histoire : «[...] à partir de cette confession, il n'y a plus eu de discussion sur le sujet en Argentine. [...] Les lois du pardon empêchaient de juger les responsables, mais le devoir de l'État d'enquêter sur la vérité et de la communiquer aux familles demeurait. C'est ainsi que les jugements pour la vérité ont commencé [...].» (Verbitsky, 2008, p.17.) Il en va de même en Colombie, où les confessions des paramilitaires devant les tribunaux ne laissent planer aucun doute sur la réalité des massacres qui leur étaient attribués jusqu'à présent: "Comme le droit international parle de massacre au-delà de trois morts, il ne fallait pas qu'on nous attribue autant de massacres, alors nous avons utilisé des serpents et ces morts apparaissaient comme des accidents de la nature9."

Le juge Baltasar Garzón a déclaré la nullité des lois de punto final en vertu du concept que si un crime contre l'humanité n'est pas puni là où il a été commis, il peut l'être partout ailleurs dans le monde, car le bien juridique à protéger, c'est le genre humain. Ce précédent représente un espoir pour la Colombie :

Il existe un moment historique pour chaque chose. Une loi n'est pas quelque chose de définitif [...]; elle exprime seulement des rapports de forces à un moment déterminé, qui peuvent se modifier demain. La lutte pour la vérité et la justice ne doivent donc pas cesser parce que les conditions du moment sont défavorables. Cette bataille ne se livre pas devant les tribunaux, mais dans la société et son degré de conscience. (Verbitsky, 2008, p. 18.)

Dans ce contexte, l'écriture testimoniale convoque la société colombienne comme juge et témoin et favorise une reconstruction de l'espace public. Le fait de témoigner sousentend en effet un domaine partagé, dans lequel se débattent les questions de morale, de jugement, de sens commun. C'est rendre toute sa valeur au champ du jugement. Le verbe juger renvoie en effet à une faculté individuelle, mais aussi à la structure dynamique du rapport entre les humains, car il fixe "[...] la spécificité de cette espèce particulière d'êtres dont l'organisation sociale s'édifie sur la possibilité et l'obligation de donner sens au monde » (Dulong, 1998, p. 126). La multiplicité de points de vue contenue dans ces récits à la première personne les porte à la lumière de l'espace public où ils révèlent tous leurs aspects et deviennent objets de discussions entre les uns et les autres, «[c]ar la condition de cette multiplicité de perspectives, c'est la présence d'un espace commun, 
publico-politique, publiquement organisé, au sein duquel ces perspectives peuvent s'apparaître les unes aux autres » (Revault d'Allonnes, 2008, p. 12).

Le phénomène testimonial colombien peut finalement être envisagé comme l'ébauche d'un nouveau récit national de la violence et comme stratégie de politique participative dans la construction de la mémoire, même si cette multitude d'événements dispersés dans des micro-espaces ne se laisse pas aisément mettre en récit dans une histoire globale. La Colombie, estime D. Pécaut, n'a jamais pu élaborer « une symbologie nationale qui permette à tous de se sentir membres d'une même communauté politique " (Pécaut, 2005, p. 2). L'écriture testimoniale serait ainsi une tentative de pallier à cette carence de symbologie nationale, afin que tous puissent se sentir membres - même dans la douleur et le deuil - d'une même communauté historique. Celle-ci ne pourra s'élaborer qu'en acceptant que: "Mon passé ressemblait aux chemins de ce pays, sur lesquels une ou plusieurs croix à chaque tournant signalent le passage de la mort. » (Vásquez, 2001, p. 16.) Cette reconstruction polyphonique de l'histoire et de la mémoire se trouve en permanente construction, car elle s'effectue en plein conflit. Les médiateurs des Histoires $d u$ Conflit, par exemple, revendiquent ainsi l'aspect inachevé de ce qu'ils nomment un working progress. Cette imperfection devient méthodologie et circulation constante entre réalisation et réflexion, afin d'évaluer et de réorienter la première tout en enrichissant la seconde. Il s'agit en effet de construire "une stratégie pour produire des documents publics de conscience collective " (Franco et Rincón, 2007, p. 5), dont le processus, que l'on pourrait qualifier de sui generis, est incertain et précaire et peut aussi bien donner des fruits qu'avorter du jour au lendemain.

L'effervescence testimoniale, la testimonitis ${ }^{10}$, que connaît actuellement la Colombie montre ainsi combien il est urgent que circulent les récits, aussi insoutenables que soient certains. Si d'aucuns parlent et libèrent la parole, d'autres parleront. Quel qu'en soit le résultat, il en restera le pouvoir de raconter et la dignité de l'avoir fait.

\section{BIBLIOGRAPHIE}

ANGULo Alejandro, « Prólogo », dans A. Molano, Los Años del Tropel, Bogotá, Cinep, 1985, p. 9-19.

ARENDT Hannah, Le système totalitaire, Paris, Seuil, 1972, [1 ${ }^{\text {re }}$ éd., 1965].

BEVERLEY John et ZIMMERMAN Marc, Literatureand Politics in the central american revolutions, Austin, University of Texas Press, 1990.

CRUVELLIER Thierry, «Los medios masivos de comunicación en contextos de justicia transicional », dans Verdad, memoria histórica y medios de comunicación, Cuadernos del conflicto, Fundación Ideas Para la Paz/ Publicaciones Semana, Bogotá, 2008, p. 21-24.

DULONG Renaud, Le Témoin oculaire, Paris, Éditions de l'École des hautes études en sciences sociales, 1998. 
-, « L'émergence du témoignage historique lors de la Première Guerre mondiale », dansFrançoisCharles Gaudard et Modesta Suarez (éds), Formes discursives du témoignage, Toulouse, Éditions universitaires du Sud, 2003, p. 11-20.

FRANCO N. et RINCÓN O. (éds), Historias del conflit y reconciliación escritas por la misma gente, document communiqué par les auteurs, Bogotá, 2007.

GONZÁLEZ URIBE Guillermo, Los Niños de la guerra, Bogotá, Planeta, 2002.

GREIFF Pablo DE, «Elementos de un programa de reparaciones », dans Justicia, verdad y reparación en medio del conflicto, Cuadernos del conflicto, Fundación Ideas Para la Paz / Publicaciones Semana, Bogotá, 2005, p. 10-14.

GRUZINSKI Serge, La Pensée métisse, Paris, Fayard, 1999.

JARAMILLO Sergio, «Presentación », dans Justicia, verdad y reparación en medio del conflicto, Cuadernos del conflicto, Fundación Ideas Para la Paz/ Publicaciones Semana, Bogotá, 2005, p. 6-7. конUт Karl, « Imaginación contra barbarie », dans K. Kohut (éd.), Literatura colombiana hoy, Imaginación y barbarie, Madrid, Iberoamericana, 1994, p. 9-24.

MOLANo Alfredo et RAMíRez W., "A manera de introducción », dansA. Molano, Los Años del Tropel, Bogotá, Cinep, 1985, p. 21-33.

Molano Alfredo, El desplazamiento forzado en Colombia [en ligne], documento Internet de Médicos sin Fronteras, disponible sur <http://www.disasterinfo.net/desplazados/informes/msf/ informedesplazados_print.htm>, 24 avril 2003, 51 p.

MORENO Marvel, En diciembre llegaban las brisas, Barcelone, Plaza y Janés, 1987.

PAYNE Leigh A., « Relatos inquietantes: las confesiones de los perpetradores y los medios ", dans Verdad, memoria histórica y medios de comunicación, Cuadernos del conflicto, Fundación Ideas Para la Paz/ Publicaciones Semana, Bogotá, 2008, p. 8-15.

PÉCAUT Daniel, « Un mayor compromiso con este país » [en ligne], discurso con motivo del Doctorado Honoris causa, documento Internet, disponible sur <http:// argumentosydebates.univalle.edu.co/2000/anexo3.htm>, 5 février 2005, p. 1-5.

-, Les FARC, une guérilla sans fins ?, Paris, Lignes de repères, 2008.

PIZARRO Eduardo, « ¿Una ley para la impunidad? », document Internet, 2005, 9 p.

POSADA Gloria, Comuna 13, Medellín, texte communiqué par l'auteur, 2006, 5 p.

REVAult D'Allonnes Myriam, « Hannah Arendt et la question du mal politique », document Internet, $2008,16 \mathrm{p}$.

RICCEUR Paul, La mémoire, l'histoire, l'oubli, Paris, Éd. du Seuil, 2000.

SAlazAR Boris et CASTillo Maria, «La Hora de los dinosaurios », Bogotá, Cerec/Cidse, 2001.

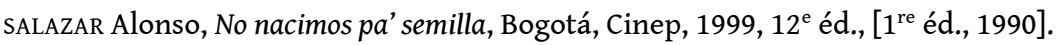

SÁNCHEZ Gonzalo, « Introducción general », dans Memoria histórica de la Comisión Nacional de Reparación y Reconciliación, Trujillo una tragedia que no cesa, Bogotá, Planeta, 2008, p. 13-29.

TOMUSCHAT Christian, « Reparación: el caso de Guatemala », dans Justicia, verdad y reparación en medio del conflicto, Cuadernos del conflicto, Fundación Ideas Para la Paz/ Publicaciones Semana, Bogotá, 2005, p. 15-17.

URIBE María Victoria, Anthropologie de l'inhumanité, Paris, Calman Lévy, 2004. 
VÁSQUEZ PERDOMO María Eugenia, Escrito para no morir, Bogotá, Coproducción ILSA, Ediciones Antropos, Ministerio de Cultura, 2001, [1 $1^{\text {re }}$ éd., 2000].

VERBITSKY Horacio, «Conférence », dans Verdad, memoria histórica y medios de comunicación, Cuadernos del conflicto, Fundación Ideas Para la Paz/ Publicaciones Semana, Bogotá, 2008, p. 16-18.

VV. AA., "Conclusiones y recomendaciones ", dans Justicia, verdad y reparación en medio del conflicto, Cuadernos del conflicto, Fundación Ideas Para la Paz/ Publicaciones Semana, Bogotá, 2005, p. 21-24.

\section{NOTES}

1. Je traduis les diverses citations de cet article.

2. Forces armées révolutionnaires colombiennes.

3. Fondateur des AUC (Autodefensas Unidas de Colombia - Autodéfenses unies de Colombie).

4. Intervention lors du symposium «Violencia, Dolor y Duelo», qui se tenait le lendemain du vote de cette loi, à Medellín.

5. Ibid.

6. Groupe de Mémoire historique, commission dépendant de la CNRR (Comisión Nacional de Reparación y Reconciliación).

7. Président de la Comisión Nacional de Reparación y Reconciliación

8. Le cas de l'Hacienda Carimagua illustre cette situation, car il est question de donner en exploitation à des multinationales de la palme africaine ce bien récupéré aux paramilitaires et de transformer les paysans spoliés en ouvriers agricoles.

9. Déclaration de « Carlos Tijeras » devant un tribunal, Bogota, El Tiempo, 4 mars 2008.

10. Selon les termes employés par l'anthropologue María Victoria Uribe.

\section{RÉSUMÉS}

Si la Colombie relève officiellement du modèle des démocraties libérales représentatives occidentales, elle s'apparente toutefois à un «laboratoire », car il s'agit d'y négocier la paix au milieu de la guerre avec des acteurs qui n'ont pas déposé les armes. Le conflit que connaît actuellement ce pays (quatre millions de déplacés, des milliers d'otages et de prisonniers, 18000 disparus) est en effet un prolongement de la violence qui y règne depuis les années quarante. Il n'y est cependant pas question de transition, car les solutions proposées par le gouvernement, loin d'ouvrir sur des possibilités de véritables négociations, suscitent de grandes controverses. La spécificité de cette situation, dans laquelle la Loi «Justice et paix » (23 juin 2005) représente un cas d'école dans le domaine de la justice transitionnelle, permettra d'apporter des éléments dans l'étude de cette dernière, à la lumière de l'abondante circulation de récits à la première personne qui s'observe aujourd'hui dans le pays.

If Colombia recovers officially from the model of the western representative liberal democracies, it is however similar to a "laboratory", because it is a question of negotiating the peace in the middle of the war with actors there who did not lay down arms. The conflict which knows at 
present this country (four millions of moved, thousands of hostages and prisoners, 18000 missing persons) is indeed a continuation of the violence which reigns there since the forties. It is however no doubt transition there, because the solutions proposed by the government, far from opening on possibilities of real negotiations, arouse big controversies.

The specificity of this situation, in which the Law "Justice and peace" (23 June 2005) represents a textbook case in the field of the transitional justice, will allow to bring elements in the study of this last one, in the light of plentiful circulation of narratives to the first person which is observed in this country today.

INDEX

Keywords : Colombia, conflict, justice, testimony, transition

Mots-clés : Colombie, conflit, justice, témoignage, transition

\section{AUTEUR}

\section{MARIE ESTRIPEAUT-BOURJAC}

Maître de conférences, IUFM, université Montesquieu-Bordeaux 4, membre de LAPRIL

(Littérature, Arts, Pluridisciplinarité, Représentations, Imaginaire, Langages) 